T. Watanabe

Nagoya Math. J.

Vol. 132 (1993), 141-153

\title{
OSCILLATION OF MODES OF SOME SEMI-STABLE LÉVY PROCESSES
}

\author{
TOSHIRO WATANABE
}

\section{\$1. Introduction}

In this paper it is shown that there is a unimodal Lévy process with oscillating mode. After the author first constructed an example of such a selfdecomposable process, Sato pointed out that it belongs to the class of semi-stable processes with $\beta<0$. We prove that all non-symmetric semi-stable selfdecomposable processes with $\beta<0$ have oscillating modes.

A measure $\mu$ on $\mathbf{R}$ is said to be unimodal with mode $a \in \mathbf{R}$ if $\mu(d x)=$ $c \delta_{a}(d x)+f(x) d x$, where $c$ is non-negative, $\delta_{a}$ is the delta measure at $a$ and $f(x)$ is non-decreasing on $(-\infty, a)$ and non-increasing on $(a, \infty)$. If a measure $\mu$ is unimodal, then either its mode is unique or the set of its modes is a closed interval. Let $\left\{X_{t}\right\}, t \in[0, \infty)$, be a Lévy process on $\mathbf{R}$ (that is, a stochastically continuous process with stationary independent increments starting at the origin) and let $\mu_{t}$ be the distribution of $X_{t}$. The Lévy process $\left\{X_{t}\right\}$ is said to be unimodal if $\mu_{t}$ is unimodal for each $t$. When a Lévy process $\left\{X_{t}\right\}$ is unimodal, we denote a mode of $\mu_{t}$ by $a(t)$. In case the set of modes of $\mu_{t}$ is a closed interval, there is freedom of choice of $a(t)$. The Lévy process $\left\{X_{t}\right\}$ is said to be self-decomposable if $\mu_{t}$ is an $L$ distribution for each $t$. A self-decomposable Lévy process is simply called a self-decomposable process. Yamazato proves in the celebrated paper [16] that every self-decomposable process is unimodal. We say that a Lévy process $\left\{X_{t}\right\}$ is semi-stable if there exist real numbers $\beta$ and $\gamma$ such that $0<|\beta|<1,1<\gamma$, $\gamma=|\beta|^{-\lambda}(0<\lambda \leqq 2)$ and

$$
\hat{\mu}_{t}(z)=\hat{\mu}_{r t}(\beta z)
$$

for every $z \in \mathbf{R}$ and every $t \geqq 0$, where

$$
\hat{\mu}_{t}(z)=\int_{0}^{\infty} e^{i z x} \mu_{t}(d x) .
$$

Received June 22, 1992. 
Semi-stable processes are introduced by Lévy [2].

Many results on unimodality of Lévy processes are obtained by Medgyessy [3], Sato [4, 5, 6], Sato-Yamazato [7], Steutel-van Harn [8], Watanabe [9, 10, 11, $12,13]$, Wolfe $[14,15]$ and Yamazato $[16,17,18,19,20]$. Among these works, only Sato $[4,5,6]$ investigates behavior of modes of unimodal Lévy processes. He shows in [4] that if a unimodal Lévy process $\left\{X_{t}\right\}$ has mean $m=E X_{1}(-\infty \leqq$ $m \leqq \infty)$, then

$$
\lim _{t \rightarrow \infty} t^{-1} a(t)=m
$$

Hence $a(t) \rightarrow \infty$ in case $0<m \leqq \infty$ and $a(t) \rightarrow-\infty$ in case $-\infty \leqq m<0$, as $t \rightarrow \infty$. The purpose of this paper is to show that a unimodal Lévy process $\left\{X_{t}\right\}$ can have mode $a(t)$ oscillating as $t \rightarrow \infty$ if $m=0$ or if $m$ does not exist. Namely we shall prove the following theorem.

THEOREM 1. Let $\left\{X_{t}\right\}$ be a non-symmetric semi-stable self-decomposable process with $-1<\beta<0$ and $0<\lambda<2$. Then $a(t)$ is unique for each $t \geqq 0$, continuous on $[0, \infty)$ and oscillating as $t \rightarrow \infty$ and $t \downarrow 0$ :

$$
\begin{aligned}
& \limsup _{t \rightarrow \infty} a(t)=\infty, \quad \liminf _{t \rightarrow \infty} a(t)=-\infty . \\
& \lim \sup \operatorname{sgn} a(t)=1, \quad \lim \inf \operatorname{sgn} a(t)=-1 . \\
& t \downarrow 0 \quad t \downarrow 0
\end{aligned}
$$

Moreover, if $0<\lambda<1$, then

$$
\limsup _{t \rightarrow \infty} t^{-1} a(t)=\infty \text { and } \liminf _{t \rightarrow \infty} t^{-1} a(t)=-\infty .
$$

Acknowledgements. The author should like to express his gratitude to Professor K. Sato who carefully read the manuscript and gave helpful advice. He is also grateful to Professor M. Yamazato for valuable comments.

\section{§2. Restatement of Theorem 1}

Let $\left\{X_{t}\right\}$ be a Lévy process on $\mathbf{R}$. Then the characteristic function of $X_{t}$ is expressed as

(2.1) $E \exp \left(i z X_{t}\right)=\exp (t \psi(z))$

(2.2) $\phi(z)=i b z-2^{-1} \sigma^{2} z^{2}+\int_{-\infty}^{\infty}\left(e^{i z x}-1-i z x\left(1+x^{2}\right)^{-1}\right) \nu(d x)$, 
where $b \in \mathbf{R}, \sigma^{2} \geqq 0$ and $\nu$ is a measure on $\mathbf{R}$ with $\nu(\{0\})=0$ and $\int_{-\infty}^{\infty} x^{2}\left(1+x^{2}\right)^{-1} \nu(d x)<\infty$, called the Lévy measure of $\left\{X_{t}\right\}$. We define $k(x)$ by $\nu(d x)=|x|^{-1} k(x) d x$, if $\nu$ is absolutely continuous. A necessary and sufficient condition for a Lévy process $\left\{X_{t}\right\}$ to be self-decomposable is that $\nu$ is absolutely continuous and $k(x)$ is non-decreasing on $(-\infty, 0)$ and non-increasing on $(0, \infty)$.

Let $\left\{X_{t}\right\}$ be a semi-stable Lévy process with $-1<\beta<0$ and $0<\lambda<2$. Then $\nu$ is given by

$$
\begin{gathered}
\int_{-\infty}^{u-} \nu(d x)=|u|^{-\lambda} \xi(\log |u|) \text { for } u<0, \\
\int_{u+}^{\infty} \nu(d x)=u^{-\lambda} \xi(\log u-\log |\beta|) \text { for } u>0,
\end{gathered}
$$

where $\xi(x)$ is a positive right-continuous periodic function on $R$ with period $-2 \log |\beta|$. Further $\phi(z)$ defined in (2.1) is represented as follows:

$$
\phi(z)=\int_{-\infty}^{\infty}\left(e^{i z x}-1\right) \nu(d x)
$$

for $0<\lambda<1$,

$$
\phi(z)=\int_{-\infty}^{\infty}\left(e^{i z x}-1-i z x\right) \nu(d x)
$$

for $1<\lambda<2$, and

$$
\psi(z)=i b z+\int_{-\infty}^{\infty}\left(e^{i z x}-1-i z x\left(1+x^{2}\right)^{-1}\right) \nu(d x)
$$

with

$$
2 b+\int_{-\infty}^{\infty} \frac{\left(1-\beta^{2}\right) x^{3}}{\left(1+x^{2}\right)\left(1+\beta^{2} x^{2}\right)} \nu(d x)=0
$$

for $\lambda=1$. Conversely these are sufficient conditions for a Lévy process $\left\{X_{t}\right\}$ to be semi-stable with $-1<\beta<0$ and $0<\lambda<2$. This is easily proved by using the discussion of Kagan-Linnik-Rao [1]. Note that $E\left|X_{1}\right|=\infty$ for $0<\lambda \leqq 1$ and $E X_{1}=0$ for $1<\lambda<2$. Thus a Lévy process $\left\{X_{t}\right\}$ is self-decomposable and semi-stable with $-1<\beta<0$ and $0<\lambda<2$ if and only if the following conditions are satisfied:

$(\mathrm{S} .1) \nu$ is represented as 
(2.8)

$$
\begin{aligned}
\nu(d x) & =|x|^{-\lambda-1} \eta(\log |x|) d x & & \text { for } x<0 \\
& =x^{-\lambda-1} \eta(\log x-\log |\beta|) d x & & \text { for } x>0
\end{aligned}
$$

where $\eta(x)$ is a positive right-continuous periodic function on $\mathbf{R}$ with period $-2 \log |\beta|$.

(S.2) $\exp (-\lambda x) \eta(x)$ is non-increasing on $\mathbf{R}$.

(S.3) The equation (2.4), (2.5), or (2.6) with (2.7) holds according as $0<\lambda<1$, $1<\lambda<2$, or $\lambda=1$.

In general there are two possible cases for a unimodal Lévy process $\left\{X_{t}\right\}$ :

Case 1. For each $t$ zero is a mode of $\mu_{t}$.

Case 2. For some $t_{0}$ zero is not a mode of $\mu_{t_{0}}$.

Let $\left\{X_{t}\right\}$ be a semi-stable self-decomposable process with $-1<\beta<0$ and $0<\lambda<2$. Since $\left\{X_{t}\right\}$ is self-decomposable, $\mu_{t}$ is absolutely continuous and unimodal for each $t>0$. Let $\mu_{t}(d x)=f_{t}(x) d x$ for $t>0$. We find from the representation (2.8) of $\nu$ that $a(t)$ is unique for each $t \geqq 0$ by Theorem 1.3 of Sato-Yamazato [7] and hence $a(t)$ is continuous on $[0, \infty)$ by Lemma 2.1 of Sato [5]. We see from semi-stability that

$$
f_{r t}(x)=|\beta| f_{t}(\beta x),
$$

which implies that

$$
a(\gamma t)=\beta^{-1} a(t)
$$

Repeating this procedure, we find that

$$
a\left(\gamma^{n} t\right)=\beta^{-n} a(t)
$$

for every integer $n$. Hence if $\left\{X_{t}\right\}$ is in Case 2, then $a\left(\gamma^{n} t_{0}\right)$ is oscillating as $n \rightarrow \infty$ and sgn $a\left(\gamma^{n} t_{0}\right)$ is oscillating as $n \rightarrow-\infty$ and satisfies (1.4). That is, $a(t)$ is continuous on $[0, \infty)$ and oscillating as $t \rightarrow \infty$ and sgn $a(t)$ is oscillating as $t \downarrow 0$. Moreover, if $0<\lambda<1$, then

$$
\frac{a\left(\gamma^{n} t_{0}\right)}{\gamma^{n} t_{0}}=\frac{a\left(t_{0}\right)}{t_{0}(\gamma \beta)^{n}}
$$

with $|\beta \gamma|=|\beta|^{1-\lambda}<1$ and hence $t^{-1} a(t)$ is oscillating as $t \rightarrow \infty$ and satisfies (1.5). Thus if we show the following theorem, then Theorem 1 is true. 
THEOREM $1^{\prime}$. Let $\left\{X_{t}\right\}$ be a semi-stable self-decomposable process with $-1<\beta$ $<0$ and $0<\lambda<2$. If $\left\{X_{t}\right\}$ is non-symmetric, then it is in Case 2.

Let us denote by $\operatorname{Re} w$ and $\operatorname{Im} w$ the real part and the imaginary part of a complex number $w$, respectively.

We see from (1.1) and (2.1) that every non-symmetric semi-stable process with $-1<\beta<0$ satisfies the following balancing condition:

(B) There exist positive numbers $\theta_{1}$ and $\theta_{2}$ such that $\theta_{2}>\theta_{1}, \operatorname{Im} \phi\left(\theta_{1}\right) \neq 0$ and $\operatorname{Im} \phi\left(\theta_{2}\right)=0$.

In fact, there exists $\theta_{1}>0$ such that $\operatorname{Im} \phi\left(\theta_{1}\right) \neq 0$, since the process is non-symmetric. Note that $\operatorname{Im} \phi(z)$ is a continuous odd function. Hence, from semi-stability with $-1<\beta<0, \operatorname{Im} \phi\left(|\beta|^{-1} \theta_{1}\right)=-\gamma \operatorname{Im} \phi\left(\theta_{1}\right)$, which yields the existence of $\theta_{2}$ such that $|\beta|^{-1} \theta_{1}>\theta_{2}>\theta_{1}$ and $\operatorname{Im} \phi\left(\theta_{2}\right)=0$.

In Section 3 we shall prove the following theorem, which is a generalization of Theorem $1^{\prime}$.

Theorem 2. Let $\left\{X_{t}\right\}$ be a self-decomposable process satisfying (B). Then $\left\{X_{t}\right\}$ is in Case 2.

\section{§3. Proof of Theorem 2}

In order to prove Theorem 2, we need several lemmas. A Lévy process is said to be non-deterministic, if it is not a deterministic motion.

Lemma 3.1. Let $\left\{X_{t}\right\}$ be a non-deterministic self-decomposable process. Then we have

( i ) $\operatorname{Re} \phi(z)$ is a continuous even function on $\mathbf{R}$ and $-\operatorname{Re} \phi(z)$ is positive and increasing on $(0, \infty)$ satisfying $\operatorname{Re} \phi(0)=0$ and $\lim _{z \rightarrow \infty}-\operatorname{Re} \phi(z)=\infty$.

(ii) $\operatorname{Im} \phi(z)$ is a continuous odd function on $\mathbf{R}$.

Proof. We shall only prove that $-\operatorname{Re} \phi(z)$ is increasing on $(0, \infty)$, since the other assertions are trivial. We obtain from (2.2) that

$$
-\operatorname{Re} \psi(z)=2^{-1} \sigma^{2} z^{2}+\int_{0}^{\infty}(1-\cos z x) x^{-1} h(x) d x,
$$

where $h(x)=k(x)+k(-x)$ is non-increasing on $(0, \infty)$ by self-decomposability. Let $0<z_{1}<z_{2}$. We have 


$$
\begin{aligned}
& -\operatorname{Re} \phi\left(z_{2}\right)+\operatorname{Re} \phi\left(z_{1}\right) \\
& =2^{-1} \sigma^{2}\left(z_{2}^{2}-z_{1}^{2}\right)+\int_{0}^{\infty}(1-\cos u) u^{-1}\left(h\left(\frac{u}{z_{2}}\right)-h\left(\frac{u}{z_{1}}\right)\right) d u \geqq 0 .
\end{aligned}
$$

In (3.2) the equality “ $=0$ ” holds if and only if

$$
\sigma=0 \text { and } h\left(\frac{x}{z_{2}}\right)=h\left(\frac{x}{z_{1}}\right) \text { for every } x>0,
$$

since we can assume that $h(x)$ is right-continuous on $(0, \infty)$. The condition (3.3) shows that, for every $x>0$,

$$
h(x)=h\left(\left(\frac{z_{2}}{z_{1}}\right)^{n} x\right) \rightarrow 0
$$

as $n \rightarrow \infty$, which yields $\nu=0$. Therefore, the equality " $=0$ " in (3.2) does not hold, since $\left\{X_{t}\right\}$ is non-deterministic. Thus we have proved Lemma 3.1.

Lemma 3.2. Let $\left\{X_{t}\right\}$ be a non-deterministic self-decomposable process. Then, for every $z_{1} \in \mathbf{R}$, there exist positive numbers $c\left(z_{1}\right)$ and $\delta\left(z_{1}\right)$ such that

$$
\left|\operatorname{Re} \phi(z)-\operatorname{Re} \phi\left(z_{1}\right)\right| \geqq c\left(z_{1}\right)\left|z-z_{1}\right|^{3}
$$

for all $z$ satisfying $\left|z-z_{1}\right| \leqq \delta\left(z_{1}\right)$.

Proof. Suppose that $\sigma^{2}>0$. Then we find from (3.2) that

$$
\left|\operatorname{Re} \phi(z)-\operatorname{Re} \phi\left(z_{1}\right)\right| \geqq 2^{-1} \sigma^{2}\left|z^{2}-z_{1}^{2}\right|
$$

for every $z_{1}$ and $z$. Setting $c(0)=2^{-1} \sigma^{2}, \delta(0)=1$ and, for $z_{1} \neq 0, c\left(z_{1}\right)=$ $4^{-1} \sigma^{2}\left|z_{1}\right|$ and $\delta\left(z_{1}\right)=\left(2^{-1}\left|z_{1}\right|\right) \wedge 1$, we get (3.5). Hence, from now on, we assume that $\sigma=0$. We divide the remaining proof into two cases.

(i) Suppose that $z_{1}=0$. Then we obtain from (3.1) that

$$
-\operatorname{Re} \phi(z)=I_{1}(z)+I_{2}(z)
$$

where

$$
I_{1}(z)=\int_{0}^{\varepsilon}(1-\cos z x) x^{-1} h(x) d x
$$

and

$$
I_{2}(z)=\int_{\varepsilon}^{\infty}(1-\cos z x) x^{-1} h(x) d x
$$


for $0<\varepsilon<\infty$. Noting that $I_{2}(z) \geqq 0$, we see that

$$
\lim _{z \rightarrow 0} \frac{-\operatorname{Re} \phi(z)}{z^{2}} \geqq \lim _{z \rightarrow 0} \frac{I_{1}(z)}{z^{2}}=\int_{0}^{\varepsilon} 2^{-1} x h(x) d x>0,
$$

which implies (3.5) for sufficiently small positive numbers $c(0)$ and $\delta(0)$.

(ii) Suppose that $z_{1} \neq 0$. Without loss of generality, we can assume $z_{1}>0$. Define $h_{1}(x)=h(x)-h(x) \wedge \varepsilon$ and $h_{2}(x)=h(x) \wedge \varepsilon$ for sufficiently small $\varepsilon>0$ so that $h_{1}(x)$ does not identically vanish. Then (3.1) is expressed as

$$
-\operatorname{Re} \phi(z)=J_{1}(z)+J_{2}(z),
$$

where

$$
J_{j}(z)=\int_{0}^{\infty}(1-\cos z x) x^{-1} h_{j}(x) d x
$$

for $j=1,2$. We find from Lemma 3.1 that $J_{1}(z)$ and $J_{2}(z)$ are increasing on $(0, \infty)$. Hence

$$
\left|\operatorname{Re} \psi(z)-\operatorname{Re} \psi\left(z_{1}\right)\right| \geqq\left|J_{1}(z)-J_{1}\left(z_{1}\right)\right| .
$$

Differentiating $J_{1}(z)$, we have

$$
\begin{gathered}
\frac{d}{d z} J_{1}(z)=\int_{0}^{\infty}(\sin z x) h_{1}(x) d x \\
=z^{-1} \sum_{n=0}^{\infty} \int_{2 n \pi}^{(2 n+1) \pi}(\sin u)\left(h_{1}\left(\frac{u}{z}\right)-h_{1}\left(\frac{u+\pi}{z}\right)\right\} d u \geqq 0
\end{gathered}
$$

for $z>0$, because $h_{1}(x)$ is non-increasing on $(0, \infty)$. If $(d / d z) J_{1}\left(z_{1}\right)>0$, then (3.5) follows from (3.10) for sufficiently small positive numbers $c\left(z_{1}\right)$ and $\delta\left(z_{1}\right)$. Suppose that $(d / d z) J_{1}\left(z_{1}\right)=0$. We find from (3.11) that $(d / d z) J_{1}\left(z_{1}\right)=0$ if and only if

$$
h_{1}\left(\frac{2 n \pi}{z_{1}}+\right)=h_{1}\left(\frac{2(n+1) \pi}{z_{1}}-\right)
$$

for every non-negative integer $n$, that is, $h_{1}(x)$ is written as

$$
h_{1}(x)=\sum_{j=1}^{N} \varepsilon_{j} I_{\left(0, b_{j}\right)}(x) \text {, }
$$

for $x>0$, where $N$ is a positive integer and, for each $j, \varepsilon_{j}$ is a positive number, $b_{j}$ $=z_{1}^{-1} 2 n_{j} \pi$ for some positive integer $n_{j}$ and $I_{\left(0, b_{j}\right)}(x)$ is the indicator function of the interval $\left(0, b_{j}\right)$. We obtain from (3.13) that 


$$
\frac{d}{d z} J_{1}(z)=\sum_{j=1}^{N} \varepsilon_{j} z^{-1}\left(1-\cos z b_{j}\right)
$$

Differentiating (3.14) and then letting $z=z_{1}$,

$$
\frac{d^{2}}{d z^{2}} J_{1}\left(z_{1}\right)=\sum_{j=1}^{N} \varepsilon_{j}\left\{-z_{1}^{-2}\left(1-\cos z_{1} b_{j}\right)+z_{1}^{-1} b_{j} \sin z_{1} b_{j}\right\}=0
$$

and

$$
\begin{aligned}
\frac{d^{3}}{d z^{3}} J_{1}\left(z_{1}\right)= & \sum_{j=1}^{N} \varepsilon_{j}\left\{2 z_{1}^{-3}\left(1-\cos z_{1} b_{j}\right)-2 z_{1}^{-2} b_{j} \sin z_{1} b_{j}\right. \\
& \left.\quad+z_{1}^{-1} b_{j}^{2} \cos z_{1} b_{j}\right\} \\
= & \sum_{j=1}^{N} \varepsilon_{j} z_{1}^{-1} b_{j}^{2}>0 .
\end{aligned}
$$

These show that (3.5) is true for $z_{1}>0$ with sufficiently small positive numbers $c\left(z_{1}\right)$ and $\delta\left(z_{1}\right)$ when $(d / d z) J_{1}\left(z_{1}\right)=0$. The proof of Lemma 3.2 is complete.

Let us denote the complex plane by $\mathbf{C}$.

Lemma 3.3. Let $\left\{X_{t}\right\}$ be a non-deterministic self-decomposable process. Suppose that $\left\{X_{t}\right\}$ is in Case 1. Let $c_{1}=2 / h(0+)$ if $\sigma=0$ and $0<h(0+)<\infty$. Let $c_{1}=0$ if $h(0+)=\infty$ or if $\sigma^{2}>0$. Let

$$
D=\left\{\bigcup_{z \geqq 0} L_{z}\right\} \cup\{w \in \mathbf{C}: \operatorname{Re} w<0\}
$$

with $L_{z}=\{w \in \mathbf{C}: w=-\operatorname{Re} \phi(z)+y i,|y|>|\operatorname{Im} \phi(z)|\}$, that is, $D$ is the connected component containing -1 of the set $\mathbf{C} \cap\{-\phi(z): z \in \mathbf{R}\}^{c}$. Then

$$
\int_{-\infty}^{\infty} \frac{z \alpha \exp [c\{\alpha+\phi(z)\}]}{\alpha+\phi(z)} \mathrm{d} z=0
$$

for every $c>c_{1}$ and $\alpha \in D$.

Proof. From Lemma 2.4 of Sato-Yamazato [7], we find that $|z \exp (t \phi(z))|$ is integrable on $\mathbf{R}$ with respect to $z$ for $t>c_{1}$. Hence the density function $f_{t}(x)$ of $\mu_{t}(d x)$ is continuously differentiable in $x$ for $t>c_{1}$. Since $\left\{X_{t}\right\}$ is in Case 1 ,

$$
\frac{d}{d x} f_{t}(0)=\frac{-i}{2 \pi} \int_{-\infty}^{\infty} z \exp (t \phi(z)) d z=0
$$

for $t>c_{1}$. We have 


$$
\int_{C}^{\infty}|z \exp [t\{\alpha+\phi(z)\}]| d t=-\frac{|z| \exp [c\{\operatorname{Re} \alpha+\operatorname{Re} \phi(z)\}]}{\operatorname{Re} \alpha+\operatorname{Re} \psi(z)}
$$

which is integrable on $\mathbf{R}$ with respect to $z$ for $c>c_{1}$ and $\operatorname{Re} \alpha<0$. By using Fubini's theorem, we obtain from (3.19) that

$$
\begin{aligned}
0 & =\int_{c}^{\infty} d t \int_{-\infty}^{\infty} z \exp [t\{\alpha+\phi(z)\}] d z \\
& =-\int_{-\infty}^{\infty} \frac{z \exp [c\{\alpha+\phi(z)\}]}{\alpha+\phi(z)} d z
\end{aligned}
$$

for $c>c_{1}$ and $\operatorname{Re} \alpha<0$. Define

$$
F(\alpha)=\int_{-\infty}^{\infty} \frac{z \exp [c\{\alpha+\phi(z)\}]}{\alpha+\phi(z)} d z
$$

and

$$
F_{N}(\alpha)=\int_{-N}^{N} \frac{z \exp [c\{\alpha+\phi(z)\}]}{\alpha+\phi(z)} d z
$$

for $c>c_{1}, \alpha \in D$ and $N>0$. We note from Lemma 3.1 that $D$ is a domain in $\mathbf{C}$ containing the left half plane. Because $F_{N}(\alpha)$ is analytic in $D$ with respect to $\alpha$ and convergent to $F(\alpha)$ uniformly on every compact set in $D$ as $N \rightarrow \infty, F(\alpha)$ is analytic in $D$. We see from (3.21) that $F(\alpha)=0$ for $\operatorname{Re} \alpha<0$ and hence $F(\alpha)=$ 0 in $D$ by the uniqueness principle. Multiplying $\alpha$ to the equation $F(\alpha)=0$, we get (3.18). Thus we have proved Lemma 3.3.

Proof of Theorem 2. We find from (B) that $\left\{X_{t}\right\}$ is non-symmetric and non-deterministic. Suppose that $\left\{X_{t}\right\}$ is in Case 1 . We shall show that this leads to a contradiction. Without loss of generality, we can assume from (B) that there exist real numbers $z_{1}$ and $z_{2}$ such that $0 \leqq z_{1}<z_{2}, \operatorname{Im} \phi\left(z_{1}\right)=\operatorname{Im} \phi\left(z_{2}\right)=0$ and $\operatorname{Im} \phi(z)<0$ on $\left(z_{1}, z_{2}\right)$. Define

$$
g(\alpha, c, z)=\frac{z \alpha \exp [c\{\alpha+\phi(z)\}]}{\alpha+\phi(z)} .
$$

Let $\varepsilon$ and $\delta$ be sufficiently small positive numbers. Let

$E(\delta, 1)=\left\{z \in \mathbf{R}: z_{1}-\delta \leqq|z| \leqq z_{1}+\delta\right\}$,

$E(\delta, 2)=\left\{z \in \mathbf{R}: z_{2}-\delta \leqq|z| \leqq z_{2}+\delta\right\}$,

$E(\delta, 3)=\left\{z \in \mathbf{R}: z_{1}+\delta \leqq|z| \leqq z_{2}-\delta\right\}$ and

$E(\delta, 4)=\left\{z \in \mathbf{R}:|z| \leqq z_{1}-\delta\right.$ or $\left.|z| \geqq z_{2}+\delta\right\}$. Then we have 


$$
\int_{-\infty}^{\infty} g(\alpha, c, z) d z=\sum_{j=1}^{4} I_{j}(\alpha, c, \delta),
$$

where $I_{j}(\alpha, c, \delta)=\int_{E(\delta, j)} g(\alpha, c, z) d z$ for $1 \leqq j \leqq 4$. For complex numbers $w_{1}$ and $w_{2}$ let us denote by $L\left(w_{1}, w_{2}\right)$ the directed line-segment from $w_{1}$ to $w_{2}$ in $\mathbf{C}$. Let $K=\sup _{z_{1}<z<z_{2}}(-2 \operatorname{Im} \phi(z))$,

$$
\begin{aligned}
& \Gamma(\varepsilon, 1)=L\left(-\phi\left(z_{1}\right)-\varepsilon i,-\phi\left(z_{1}\right)-K i\right), \\
& \Gamma(\varepsilon, 2)=L\left(-\phi\left(z_{1}\right)-K i,-\phi\left(z_{2}\right)-K i\right), \\
& \Gamma(\varepsilon, 3)=L\left(-\phi\left(z_{2}\right)-K i,-\phi\left(z_{2}\right)-\varepsilon i\right), \\
& \Gamma(\varepsilon, 4)=L\left(-\phi\left(z_{2}\right)+\varepsilon i,-\phi\left(z_{2}\right)+K i\right), \\
& \Gamma(\varepsilon, 5)=L\left(-\phi\left(z_{2}\right)+K i,-\phi\left(z_{1}\right)+K i\right), \\
& \Gamma(\varepsilon, 6)=L\left(-\phi\left(z_{1}\right)+K i,-\phi\left(z_{1}\right)+\varepsilon i\right),
\end{aligned}
$$

and let $\Gamma(\varepsilon)$ be the union of the directed line-segments $\Gamma(\varepsilon, j), j=1, \ldots, 6$. In the following, integrals along $\Gamma(\varepsilon, j)$ or $\Gamma(\varepsilon)$ with respect to $\alpha$ are line integrals. Note that $\Gamma(\varepsilon)$ is contained in $D$ by Lemma 3.1. Hence we obtain from (3.18) in Lemma 3.3 that

$$
\int_{\Gamma(\varepsilon)} d \alpha \int_{-\infty}^{\infty} g(\alpha, c, z) d z=0
$$

for $0<\varepsilon<K$ and for $c>c_{1}$. Let $A(\varepsilon)$ be the union of the directed line-segments $\Gamma(\varepsilon, j), j=2, \ldots, 5$, and let $B(\varepsilon)$ be the union of $\Gamma(\varepsilon, 1)$ and $\Gamma(\varepsilon, 6)$. Let $\tilde{A}(\varepsilon)$ and $\tilde{B}(\varepsilon)$ denote the sets of points on $A(\varepsilon)$ and $B(\varepsilon)$, respectively. By Lemma 3.1 , we can choose sufficiently small positive numbers $\delta_{1}$ and $d_{1}$, which do not depend on $\varepsilon$, such that

$$
|\alpha+\phi(z)| \geqq d_{1}
$$

for $z \in E\left(\delta_{1}, 1\right)$ and $\alpha \in \tilde{A}(\varepsilon)$. Hence we can find $M_{1}>0$, which does not depend on $\varepsilon$, such that

$$
|g(\alpha, c, z)| \leqq M_{1}
$$

for $z \in E\left(\delta_{1}, 1\right)$ and $\alpha \in \tilde{A}(\varepsilon)$. It follows that

$$
\begin{aligned}
& \lim _{\delta \rightarrow 0} \lim _{\varepsilon \rightarrow 0} \int_{A(\varepsilon)} I_{1}(\alpha, c, \delta) d \alpha \\
= & \lim _{\delta \rightarrow 0} \lim _{\varepsilon \rightarrow 0} \int_{E(\delta, 1)} d z \int_{A(\varepsilon)} g(\alpha, c, z) d \alpha=0 .
\end{aligned}
$$

On the other hand, we can choose $\delta_{2}>0$ and $M_{2}>0$, which do not depend on $\varepsilon$, 
such that

$$
|g(\alpha, c, z)(\alpha+\phi(z))| \leqq M_{2}
$$

for $z \in E\left(\delta_{2}, 1\right)$ and $\alpha \in \tilde{B}(\varepsilon)$. Hence we have, for $0<\delta<\delta_{2}$,

$$
\left|\int_{B(\varepsilon)} I_{1}(\alpha, c, \delta) d \alpha\right| \leqq M_{2} \int_{E(\delta, 1)} d z \int_{B(\varepsilon)} \frac{|d \alpha|}{|\alpha+\phi(z)|}
$$

Define $\quad N=\sup _{z \in E\left(\delta_{2}, 1\right)}|\operatorname{Im} \phi(z)|, L=\sup _{z \in E\left(\delta_{2}, 1\right)}\left|\operatorname{Re} \phi(z)-\operatorname{Re} \phi\left(z_{1}\right)\right| \quad$ and $a=\left|\operatorname{Re} \phi(z)-\operatorname{Re} \phi\left(z_{1}\right)\right|^{-1}(K+N)$. For $z \in E\left(\delta_{2}, 1\right), z \neq z_{1}$, we get that

$$
\begin{aligned}
& \int_{B(\varepsilon)} \frac{|d \alpha|}{|\alpha+\phi(z)|} \\
= & \int_{\varepsilon}^{K}\left[\left\{\left(\operatorname{Re} \phi(z)-\operatorname{Re} \phi\left(z_{1}\right)\right)^{2}+(\operatorname{Im} \phi(z)-\theta)^{2}\right\}^{-1 / 2}\right. \\
& \left.+\left\{\left(\operatorname{Re} \phi(z)-\operatorname{Re} \phi\left(z_{1}\right)\right)^{2}+(\operatorname{Im} \phi(z)+\theta)^{2}\right\}^{-1 / 2}\right] d \theta \\
< & 8 \int_{0}^{a}(1+u)^{-1} d u \\
\leqq & 8 \log (K+N+L)-8 \log \left|\operatorname{Re} \phi(z)-\operatorname{Re} \phi\left(z_{1}\right)\right|,
\end{aligned}
$$

where we use $\left(1+u^{2}\right)^{-1 / 2} \leqq 2(1+u)^{-1}$ for $u \geqq 0$. Recalling Lemma 3.2 , we obtain from (3.31) and (3.32) that

$$
\begin{aligned}
& \lim _{\delta \rightarrow 0} \lim _{\varepsilon \rightarrow 0} \int_{B(\varepsilon)} I_{1}(\alpha, c, \delta) d \alpha \\
= & \lim _{\delta \rightarrow 0} \lim _{\varepsilon \rightarrow 0} \int_{E(\delta, 1)} d z \int_{B(\varepsilon)} g(\alpha, c, z) d \alpha=0 .
\end{aligned}
$$

Hence we find from (3.29) that

$$
\lim _{\delta \rightarrow 0} \lim _{\varepsilon \rightarrow 0} \int_{\Gamma(\varepsilon)} I_{1}(\alpha, c, \delta) d \alpha=0 .
$$

Similarly we get that

$$
\lim _{\delta \rightarrow 0} \lim _{\varepsilon \rightarrow 0} \int_{\Gamma(\varepsilon)} I_{2}(\alpha, c, \delta) d \alpha=0
$$

Making use of Cauchy's integral formula, we have

$$
\lim _{\delta \rightarrow 0} \lim _{\varepsilon \rightarrow 0} \int_{\Gamma(\varepsilon)} I_{3}(\alpha, c, \delta) d \alpha
$$




$$
\begin{aligned}
& =\lim _{\delta \rightarrow 0} 2 \pi i \int_{E(\delta, 3)} z(-\phi(z)) d z \\
& =-2 \pi i\left(\int_{z_{1}}^{z_{2}} z \phi(z) d z+\int_{-z_{2}}^{-z_{1}} z \phi(z) d z\right) \\
& =4 \pi \int_{z_{1}}^{z_{2}} z \operatorname{Im} \phi(z) d z .
\end{aligned}
$$

Since, for $c>c_{1}, I_{4}(\alpha, c, \delta)$ is analytic with respect to $\alpha$ in the rectangle $\{w$ : $\left.-\phi\left(z_{1}\right)<\operatorname{Re} w<-\phi\left(z_{2}\right),|\operatorname{Im} w|<K\right\}$, we see by Cauchy's integral theorem that

$$
\lim _{\varepsilon \rightarrow 0} \int_{\Gamma(\varepsilon)} I_{4}(\alpha, c, \delta) d \alpha=0
$$

for $c>c_{1}$. Hence we obtain from (3.26), (3.34), (3.35), (3.36) and (3.37) that

$$
\begin{aligned}
0 & =\lim _{\varepsilon \rightarrow 0} \int_{\Gamma(\varepsilon)} d \alpha \int_{-\infty}^{\infty} g(\alpha, c, z) d z \\
& =4 \pi \int_{z_{1}}^{z_{2}} z \operatorname{Im} \varphi(z) d z<0
\end{aligned}
$$

for $c>c_{1}$. This is a contradiction. Thus the proof of Theorem 2 is complete.

\section{REFERENCES}

[1] Kagan, A. M., Linnik, Yu. V. and Rao, C. R., Characterization Problems in Mathematical Statistics, John Wiley \& Sons, 1973.

[2] Lévy, P., Théorie de l'addition des variables aléatoires, 2ème éd. (1ère éd. 1937), Gauthier-Villars, Paris, 1954.

[ 3 ] Medgyessy, P., On a new class of unimodal infinitely divisible distribution functions and related topics, Studia Sci. Math. Hangar., 2 (1967), 441-446.

[4] Sato, K., Bounds of modes and unimodal processes with independent increments, Nagoya Math. J., 104 (1986), 29-42.

[5] - Behavior of modes of a class of processes with indecpendent increments, J. Math. Soc. Japan, 38 (1986), 679-695.

[6] - On unimodality and mode behavior of Lévy processes, "Probability Theory and Mathematical Statistics. Proceedings of the Sixth USSR-Japan Symposium" edited by A. N. Shiryaev et al., World Scientific, Singapore, 1992, pp. 292-305.

[ 7 ] Sato, K. and Yamazato, M., On distribution functions of class $L, Z$. Wahrsch. verw. Gebiete, 43 (1978), 273-308.

[8] Steutel, F. W. and van Harn, K., Discrete analogues of self-decomposability and stability, Ann. Probability, 7 (1979), 893-899.

[9] Watanabe, T., Non-symmetric unimodal Lévy processes that are not of class $L$, Japan. J. Math., 15 (1989), 191-203. 
[10] - On the strong unimodality of Lévy processes, Nagoya Math. J., 121 (1991), 195-199.

[11] - On unimodal Lévy processes on the nonnegative integers, J. Math. Soc. Japan, 44 (1992), 239-250.

[12] - On Yamazato's property of unimodal one-sided Lévy processes, Kodai Math. J., 15 (1992), 50-64.

[13] - Sufficient conditions for unimodality of non-symmetric Lévy processes, Kodai Math. J., 15 (1992), 82-101.

[14] Wolfe, S. J., On the unimodality of $L$ functions, Ann. Math. Statist., 42 (1971), 912-918.

[15] - On the unimodality of infinitely divisible distribution functions, Z. Wahrsch. verw. Gebiete, 45 (1978), 329-335.

[16] Yamazato, M., Unimodality of infinitely divisible distribution functions of class $L$, Ann. Probability, 6 (1978), 523-531.

[17] - On strongly unimodal infinitely divisible distributions, Ann. Probability, 10 (1982), 589-601.

[18] - Characterization of the class of upward first passage time distributions of birth and death processes and related results, J. Math. Soc. Japan, 40 (1988), $477-499$.

[19] - On subclasses of infinitely divisible distributions on $\mathrm{R}$ related to hitting time distributions of 1-dimensional generalized diffusion processes, Nagoya Math. J., 127 (1992), 175-200.

[20] - On strongly unimodal infinitely divisible distributions of class CME, Preprint.

Center for Mathematical Sciences

The University of Aizu

Ikki-machi

Aizuwakamatsu

Fukushima, 965

Japan 